\title{
Reviving external cephalic version: a review of its efficacy, safety, and technical aspects
}

\author{
Gwang Jun Kim \\ Department of Obstetrics and Gynecology, Chung-Ang University Hospital, Seoul, Korea
}

Currently, the rate of cesarean sections being performed in Korea is approximately $40 \%$, with Korea ranking 4th among the Organization for Economic Co-operation and Development countries with respect to cesarean deliveries. Breech presentation at term is an important indication for cesarean section among other factors, including medicolegal concerns and pregnancies in women of advanced maternal age. Term breech presentation is associated with a higher fetal mortality rate than that associated with a cephalic presentation. Therefore, in Korea, most of these women deliver by cesarean section to avoid the complications of vaginal breech delivery. However, cesarean section is itself associated with considerable obstetric morbidity and sometimes, mortality. External cephalic version (ECV) is a useful method to reduce the cesarean section rate in women with breech presentation and therefore to reduce the incidence of breech presentation at delivery. Studies have shown that routine use of ECV reduces the cesarean section rate by approximately two-thirds in term pregnancies with breech presentation. ECV is accepted as a safe, efficacious, and cost-effective method and is recommended by both the American College of Obstetricians and Gynecologists and the Royal College of Obstetricians and Gynecologists in all pregnancies with term breech presentation, if not contraindicated. In Korea, although most clinicians are aware of the option of ECV, their relative lack of experience in performing the procedure and fear of complications render them hesitant to perform ECV. This review is aimed at guiding obstetricians by describing the efficacy, safety concerns, and technical aspects of this procedure.

Keywords: External cephalic version; Caesarean section; Breech presentation; Cardiotocography

\section{Introduction}

Non-cephalic presentations are common in preterm pregnancies and their rate gradually decreases with increasing gestational age. Breech presentation, which is the most common non-cephalic presentation, occurs in $25 \%$ of fetuses before 28 weeks of gestation, and this rate decreases to $7 \%$ by 32 weeks of gestation and further to 3-4\% at term [1].

Vaginal breech delivery has a long history and was a common practice over the past 2 decades. In 2000, the Term Breech Trial, a large, international multicenter randomized clinical trial comparing planned vaginal deliveries with planned cesarean deliveries showed that perinatal and neonatal mortality rates, as well as serious neonatal morbidity rates were significantly higher in the planned vaginal delivery group than in the planned cesarean delivery group (16\% vs. $5 \%$ ) [2]. These findings of the Term Breech Trial significantly affected the attitude of obstetricians toward breech deliveries and since 2000, cesarean sections are being accepted as the safer option for breech delivery.

In the United States, the rate of cesarean deliveries among women in labor with breech presentation increased to 86.9\% after the Term Breech Trial [3]. Currently, in most countries including Korea, cesarean section is the usual mode of delivery for term breech presentation. This is one of the prime reasons for the current cesarean section rate in Korea being as high as $38 \%$, with Korea ranking 4th among the

Received: 2019.03.09. Revised: 2019.04.11. Accepted: 2019.05.06. Corresponding author: Gwang Jun Kim

Department of Obstetrics and Gynecology, Chung-Ang University Hospital, 102 Heukseok-ro, Dongjak-gu, Seoul 06973, Korea

E-mail: gjkim@cau.ac.kr

https://orcid.org/0000-0003-0232-0166

Articles published in Obstet Gynecol Sci are open-access, distributed under the terms of the Creative Commons Attribution Non-Commercial License (http://creativecommons. org/licenses/by-nc/3.0/) which permits unrestricted non-commercial use, distribution, and reproduction in any medium, provided the original work is properly cited.

Copyright $\odot 2019$ Korean Society of Obstetrics and Gynecology 


\section{Obstetrics \& Gynecology Science}

Vol. 62, No. 6, 2019

Organization for Economic Co-operation and Development countries with respect to cesarean deliveries [4]. Notably, other reasons include medicolegal concerns and a high percentage of pregnancies in women of advanced maternal age. This is cause for concern because at the population level, the ideal rate for cesarean sections ranges from $10 \%$ to $15 \%$, with rates $>10 \%$ not associated with reduced maternal and newborn mortality [5].

Breech presentation is the 3rd most common indication for cesarean section, after previous cesarean section and labor dystocia [6]. Approximately $12 \%$ of cesarean sections in the United States were performed in women secondary to breech presentation [7]. Although regarded as a safer mode of breech delivery by most pregnant women and clinicians alike, cesarean section is one of the most significant contributing factors to post-partum maternal morbidity rates in developed countries and is known to cause significant complications, which can sometimes result in permanent health impairment.

Thus, the rapid increase in cesarean section rates has become a global concern. With respect to breech presentation, the only option to avoid cesarean section is attempting external cephalic version (ECV), a procedure that involves rotation of the fetus from a non-cephalic to cephalic presentation by manipulating the pregnant woman's abdomen, thereby reducing the incidence of breech presentation at delivery. The routine use of this procedure has been reported to reduce the rate of cesarean sections by approximately two-thirds in term pregnancies with breech presentation [8]. The overall success rate of ECV has been reported to be $58 \%$, of which $80 \%$ women can deliver vaginally [9]. Moreover, a Cochrane systematic review reported that the use of ECV at term was associated with a clinically and statistically significant reduction in breech presentation, as well as cesarean section without significant adverse perinatal outcomes (based on Apgar scores, infant mortality, and neonatal admission rates) [1]. Furthermore, Tan et al. [8] evaluated the cost-effectiveness of ECV for term breech presentation and reported that an ECV trial was cost-effective when the probability of its success was over $32 \%$ [10].

The current recommendation by the Cochrane Foundation is to offer ECV to women with normal pregnancies and breech presentation at term (level A recommendation) [1]. Moreover, both the American College of Obstetricians and Gynecologists (ACOG) and the Royal College of Obstetricians and Gynecologists (RCOG) recommend the use of ECV as first-line management of breech presentation at term $[11,12]$.

In Korea, the birth rate is declining, with only 357,800 neonates born in 2017; however, the percentage of pregnancies among women of advanced maternal age has shown an increasing trend over the years. This perhaps explains the increasing percentage of breech presentation at term in recent times because the risk of breech presentation is known to increase in a linear manner in association with maternal age [13].

Considering an incidence rate of $5 \%$, approximately 18,000 pregnancies in Korea were associated with breech presentation at term $(5 \%$ of 357,800$)$ in 2018 . Presumably, most of these women underwent cesarean section. If an ECV trial were to be attempted in even $50 \%$ of these term breech pregnancies, it is possible that 4,500 cesarean sections could have been avoided, assuming a modest success rate of $50 \%$ (considering a cesarean section rate of $20 \%$ associated with successful ECV). This is in significant contrast to countries such as the Netherlands, where it has been reported that approximately $70 \%$ of eligible women are offered an ECV trial [14].

In Korea, low-intervention birth experience (typically referred to as "natural birth") is gaining popularity among educated pregnant women of advanced age. Thus, this is an optimal time to revive the practice of ECV. Although most clinicians are aware of ECV as a useful option, their relative lack of experience with the procedure renders them hesitant to perform ECV. Additionally, concerns over the safety of ECV exist among both clinicians as well as pregnant women [15]. Furthermore, there is a relative lack of studies and reviews regarding this topic in Korea. This review aims to encourage the revival of ECV by describing the efficacy, safety concerns, and technical aspects of this procedure.

\section{A brief history of external cephalic version}

The practice of ECV has a long history and dates back to the times of Hippocrates (460-377 BC). Aristotle (384-322 BC) is known to have described ECV, advocating that physicians advise midwives to perform this procedure [16]. Around 100 $A D$, Soranus described ECV as a method to reduce complications associated with vaginal breech birth [8], and in the 17th century, the French obstetrician Francois Mauriceau described 


\section{Obstetrics \& Gynecology Science}

Gwang Jun Kim. External cephalic version

this procedure as being "a little more difficult than turning an omelette in a frying pan" [16]. Over the last century, the practice of ECV gradually gained popularity, and by the mid1970s, it was an integral component of routine obstetric practice owing to its obvious and immediate effectiveness. However, after this period, its popularity declined owing to concerns regarding its effectiveness and safety, with reports describing considerable perinatal mortality associated with the procedure [17]. Consequently, ECV became relatively obsolete by the mid-1980s. However, after 1980, following the increasing popularity of ultrasonography and electronic fetal monitoring (EFM), the 2 ground-breaking technologies introduced in obstetrics at the time, ECV was re-established as a safe procedure [16] with an overall success rate of $65 \%$.

\section{Timing of external cephalic version}

In nulliparous women, ECV may be performed beginning at 36.0 weeks of gestation [18]. A randomized multicenter trial reported that although ECV performed at 34-36 weeks (in contrast to 37-38 weeks) decreased the rate of breech presentation at birth by $19 \%$, it was associated with an increased incidence of late preterm delivery [19]. Therefore, it is preferable to wait until 36.0 weeks to perform ECV to avoid preterm delivery. By consensus, in multipara, ECV is performed at term beginning at 37.0 weeks of gestation [11].

\section{Contraindications to external cephalic version}

ECV may not be beneficial or may in fact be harmful in a few fetal and maternal conditions. Notably, contraindications to vaginal delivery, such as placenta previa, serious fetal compromise, or major congenital anomalies serve as contraindications for ECV. Fetal well-being should be confirmed using ultrasonography and EFM prior to performing ECV.

Other contraindications for ECV are as follows:

- Onset of active labor: Uterine contractions caused by active labor make it difficult to perform an ECV trial. However, ECV can be attempted during the time interval between uterine contractions. Two studies that described intrapartum ECV reported promising results with success rates of $73 \%$ (11 of 15 attempts) [20] and 92.3\% (12 of 13 attempts) [21].
Therefore, onset of active labor is not an absolute contraindication for ECV.

- Rupture of membranes: Most clinicians regard rupture of membranes as a relative contraindication for ECV because decreased amniotic fluid volume itself hinders the trial [15]. However, ECV trials may be successfully performed in multiparous women with some amount of amniotic fluid. Thus, rupture of membranes is not an absolute contraindication for ECV.

- Vaginal bleeding: It is preferable to delay ECV or completely avoid the procedure in a woman with recent vaginal bleeding of unknown origin or bleeding secondary to placental detachment [15].

- Severe preeclampsia or eclampsia: Fetuses of women with this condition commonly develop heart rate deceleration after ECV. A previous study reported that nearly $50 \%$ of the fetuses evaluated in that study showed abnormal heart rate tracings during or immediately after the procedure [22], which suggests that although transient, ECV is a stressful event for the fetus [15].

- Multiple gestation: Twin fetuses are known to easily return to breech presentation after successful ECV. Furthermore, an ECV trial in such cases can cause rupture of the intertwin membrane, which can progress to serious complications such as amniotic band or cord entanglement. Therefore, most clinicians do not attempt ECV in women with multiple pregnancies.

- Major fetal anomalies: It is preferable to avoid ECV in women in whom prenatal ultrasonography reveals major fetal anomalies such as complex cardiac defects, significant brain malformation, and anomalies affecting the fetal pulmonary system. However, an ECV trial is not contraindicated in women in whom minor fetal anomalies are detected.

- Abnormal cardiotocography before external cephalic version: Most authors agree that ECV should not be performed in cases of abnormal EFM before the trial because the procedure may aggravate the situation.

- Growth restriction associated with an abnormal umbilical artery Doppler index: Fetuses with this condition commonly show abnormal heart rates after the ECV trial [15]. Therefore, it is safe to avoid the procedure in these cases.

- Previous cesarean section: An ECV trial can be attempted in women who wish to trial vaginal birth after previous cesarean section. This practice is based on the recommendations of a study in which ECV was performed on 42 women with 


\section{Obstetrics \& Gynecology Science}

Vol. 62, No. 6, 2019

term breech presentation with a history of previous cesarean section, with a success rate of $74.0 \%$ without significant fetal or maternal complications [23]. Similarly, another prospective, comparative cohort study reported 74 ECV trials performed on women with a history of cesarean section with a $67.1 \%$ success rate, without significant complications [24].

- Nuchal cord: No guidelines or definitive data are available regarding the risk of ECV in women with ultrasonographic evidence of nuchal cord. Based on the author's personal experience of performing $>1,000$ consecutive ECV trials (unpublished data), a single nuchal cord does not increase the risk; however, $>2$ tight nuchal cords tend to decrease the success rate of ECV.

In conclusion, as reported by a systematic review, to date, there is lack of consensus regarding the indications of ECV in pregnant women [25].

\section{Factors associated with successful external cephalic version}

The reported success rates associated with ECV vary considerably across studies ranging from $28 \%$ to $74 \%$ [26-30]. A meta-analysis showed that the success rate of ECV ranged from $16 \%$ to $100 \%$, with a pooled rate of $58 \%$ [12]. Prognostic parameters associated with the success of ECV include the following:

- Parity: Multiparous women tend to show a higher ECV success rate $(72.3 \%)$ than nulliparous women (between $40 \%$ and $64 \%)[26,28,30,31]$.

- Amniotic fluid: It has been reported that an amniotic fluid index (AFI) $>7 \mathrm{~cm}$ is associated with a successful ECV trial [26,30-32]; however, a study reported significantly higher success rates with $\mathrm{AFI}>10$ (odds ratio [OR], 1.8; 95\% confidence interval $[\mathrm{Cl}], 1.5-2.1)$ [33]. Furthermore, a systematic review described a low ECV success rate with low amniotic fluid volume, although it was not possible to define the AFI threshold associated with an unsuccessful ECV trial [34].

- Non-anterior placental location: ECV trials were more successful in women with the placenta located on the posterior uterine wall $(\mathrm{OR}, 1.9 ; 95 \% \mathrm{Cl}, 1.5-2.4)[33]$, and an anteriorly located placenta decreased the success rate of the ECV $[33,34]$. This was probably because an anterior placenta hinders palpation of the fetal head, and most clinicians do not exert full pressure when turning the fetus to avoid the risk of abruption of an anteriorly placed placenta.

- Type of breech presentation: Frank breech presentation is associated with a lower rate of success [30] than a complete breech presentation (OR, 2.3; 95\% Cl, 1.9-2.8) [33], possibly because in cases of frank breech presentation, the fetal buttocks are often engaged deeply and are firmly secured within the maternal pelvis; thus, lifting the buttocks is more difficult compared to other types of breech presentations.

- Estimated fetal weight before external cephalic version: The effect of estimated fetal weight on the success rates of ECV is controversial in that a few authors have reported greater success rates with high estimated fetal weight $[26,31]$, whereas a few others have reported no such association [35].

- Maternal body mass index (BMI): A cross-sectional analysis of 51,002 ECV trials in the United States showed lower success rates with high maternal BMI $(P<0.01)$. The success rate was $58.5 \%$ in women with $\mathrm{BMI} \geq 40 \mathrm{~kg} / \mathrm{m}^{2}$ and $65 \%$ in women with normal BMI [36].

A systematic review reported that the predictive variables for successful ECV included parity, placental location, breech engagement, and a palpable fetal head [36].

\section{Spontaneous version after external cephalic version}

In women with term gestation, reversion after successful ECV occurred in 2.2-7.5\% of cases [34,37], and spontaneous version after failed ECV occurred in $4.3 \%$ of cases and was more common in multipara [28].

\section{Cesarean section after successful external cephalic version}

Following a successful and uncomplicated ECV trial, the mean cesarean section rate ranged from $14.7 \%$ to as high as $21 \%[38,39]$. Although the pregnancy progresses beyond 40 weeks in women who undergo successful ECV, these women are known to require labor induction with a higher rate of cesarean section [40], mainly necessitated by labor dystocia $(\mathrm{OR}, 2.2 ; 95 \% \mathrm{Cl}, 1.6-3.0)$ and fetal distress (OR, 2.2; $95 \%$ $\mathrm{Cl}, 1.6-2.9)[39]$.

Parity, BMI, and amniotic fluid volume are known to affect the cesarean section rate after successful ECV 


\section{Obstetrics \& Gynecology Science}

Gwang Jun Kim. External cephalic version

$[27,28,39,41,42]$. Nulliparity increased the risk of cesarean section $(\mathrm{OR}, 2.7 ; 95 \% \mathrm{Cl}, 1.2-6.1)$ [41], with only $50 \%$ of nulliparae undergoing a vaginal vertex delivery following successful ECV, whereas the corresponding figure in multipara was as high as $88.5 \%$ [27]. A Dutch study that included 301 women reported that of all the variables evaluated, only parity could predict the risk of cesarean section [41]. Notably, only $59.5 \%$ of women with $\mathrm{BMl} \geq 40 \mathrm{~kg} / \mathrm{m}^{2}$ delivered vaginally following successful ECV; however, the corresponding figure was much higher (81.0\%) in women with normal BMI [37]. Moreover, women with AFI $\geq 13$ were significantly more likely to deliver vaginally after successful ECV [41].

Induction of labor, high BMI, and previous cesarean section were associated with an increased risk of cesarean section after successful ECV [39]. However, the time interval between ECV and delivery failed to predict spontaneous vaginal delivery after successful ECV [42]. The other variables, such as maternal age, gestational age, estimated fetal birth weight, and neonatal sex were not observed to predict cesarean section after successful ECV [42].

\section{Complications}

Although the history of ECV spanning several decades provides proof of its safety profile, this procedure continues to remain controversial. Complications of ECV are as follows:

- Transient abnormal cardiotocography patterns: With an incidence rate of 5.7-9.6\%, a transient abnormal cardiotocography pattern is the most commonly reported complication of ECV [43]. Temporary baseline bradycardia is relatively common (9-9.6\%) [44,45]; however, a report from Japan has described an exceptionally high incidence of fetal bradycardia during or immediately after ECV $(48.5 \%, 189$ of 390 cases) that lasted for $<1$ minute in $43.3 \%,<5$ minutes in $88.9 \%$, and $<10$ minutes in $98.4 \%$ of cases [22]. Bradycardia lasting $>10$ minutes occurred in 3 of 390 cases $(0.7 \%)$, and low Apgar scores at 5 minutes, with an umbilical cord arterial $\mathrm{pH}<7.1$ were observed in 2 of these cases [22].

- Fetal heart rate deceleration: Although the incidence of a persistent pathological cardiotocographic pattern was between $0.2 \%$ (2 of 980 trials) [44] and $0.37 \%$ [43] after ECV, it was not significantly associated with the AFI [32].

- Umbilical cord accidents: Success or failure of ECV was not associated with an increased risk of umbilical cord acci- dents [45].

- Placental abruption: A recent systematic review of 7,377 ECV trials reported a placental abruption incidence rate of $0.12 \%$ [43]; however, an earlier systematic review of 2,503 ECV trials published in 2006 identified no reports of placental abruption associated with ECV [46].

- Vaginal bleeding: Vaginal spotting or bleeding occurred in $0.47 \%$ of women after the procedure [43]. Although the cause of vaginal bleeding is unclear, it can infrequently be associated with placental abruption. Therefore, in the event of vaginal spotting, the ECV trial should be promptly discontinued, and close monitoring for further spotting and cardiotocography should be performed.

- Premature rupture of the membranes: A previous study has reported that immediate delivery occurred in 1.3\% of women following ECV secondary to premature rupture of membranes [47]; however, a systematic review of 2,503 ECV trials reported no such findings [46].

- Fetomaternal hemorrhage: The rate of detectable fetomaternal hemorrhage during ECV was reported to be $2.4 \%$ [48]. Moreover, estimated fetomaternal hemorrhage $>30 \mathrm{~mL}$ occurred in just 1 case $(0.08 \%)$ reported among the 1,311 ECV trials; therefore, it was recommended that Rh immunoglobulin in addition to the routine dose of 300 microgram dose need not be administered at 28 weeks of gestation and postpartum [48].

- Uterine rupture: Uterine rupture during an ECV trial is one of the most catastrophic complications. However, fortunately, this is rare, and no cases included in a systematic review of 2,503 ECV trials have reported this complication [46].

- Emergency cesarean section: The risk of emergency cesarean deliveries during and after ECV reportedly ranges between $0.2 \%$ and $0.7 \%[15,22,43,44,49]$, with the most common indication being an abnormal fetal heart rate pattern detected on EFM.

- Fetal death: A previous study reported no increase in the risk of antepartum fetal death associated with ECV [46], whereas another study reported that although no intrauterine death occurred within 24 hours of performing ECV, intrauterine death occurred in 1 case $(0.09 \%)$ at 4 weeks following an uncomplicated ECV [50]. Furthermore, a recent cohort study spanning over 18 years reported a corrected post-ECV perinatal mortality rate of $0.12 \%$ [28], whereas a systematic review of 7,377 women reported a slightly higher perinatal mortality rate of $0.16 \%$ [43]. Another Cochrane systematic 


\section{Obstetrics \& Gynecology Science}

Vol. 62, No. 6, 2019

review published in 2015 reported that perinatal mortality occurred in 2 of 644 neonates in the ECV group and in 6 of 661 neonates in the control group [1].

A meta-analysis has reported a pooled ECV complication rate of $6.1 \%$ (0.24\% for serious complications) [49]. Compared to findings in women who did not undergo ECV, ECV failure was associated with an increased risk of premature rupture of membranes (adjusted OR [aOR], 1.75; 95\% Cl, 1.60-1.90), abnormal fetal heart rate tracing (aOR, 1.78; $95 \% \mathrm{Cl}, 1.50-2.11)$, assisted ventilation at birth (aOR, 1.50; 95\% Cl, 1.27-1.78), and 5-min Apgar scores < 7 (aOR, 1.35; $95 \% \mathrm{Cl}, 1.20-1.51$ ) [51]. A large study that included 4,117 women reported that compared to expectant management, an ECV trial at term was not associated with increased prenatal morbidity or mortality [52].

Therefore, the latest guideline issued by the RCOG with respect to ECV does not recommend standard preoperative preparations for cesarean section for women undergoing ECV [18].

\section{Procedure of external cephalic version}

\section{Before performing the external cephalic version}

1) Obtaining pre-procedural informed consent Informed consent should be documented and must include the following important points:

a. Successful ECV can reduce the rate of cesarean sections (success rate approximately 60-70\%).

b. Possible use of tocolytics.

c. The procedure may be uncomfortable and occasionally painful.

d. Risks and benefits of the procedure should be explained to women/their family prior to the ECV.

\section{2) Electronic fetal monitoring}

EFM tracings should be normal before the procedure. The monitoring should continue during, as well as after the procedure.

\section{3) Ultrasonography}

Ultrasonography should be performed before ECV to confirm the fetal position and size, the type of breech, position of the fetal spine, location of the placenta, amniotic fluid vol- ume, cord around the neck, and descent of the breech fetus $[53,54]$.

\section{4) Intravenous access}

Intravenous access must be established for the administration of tocolytics, as well as for use during emergencies.

\section{Technique of external cephalic version}

After lubricating the maternal abdomen with ultrasound gel to reduce friction, real-time ultrasonography is performed intermittently during the ECV procedure to check fetal progress and to detect changes in the fetal heart rate pattern. Usually, at the first ECV attempt, the direction of rotation is a forward roll (motion in the direction of the fetal face) (Fig. 1). If this maneuver is unsuccessful, a back flip (motion in the direction of the fetal occiput) can be tried. An ECV procedure includes the following steps:

a. Dislodge the fetal buttocks from the pelvis pushing upwards and subsequently laterally with 1 hand (usually the dominant one) (Fig. 1).

b. Gently grasp the fetal head and direct it downwards with the other hand (Fig. 1).

c. Slowly rotate the fetus by pushing upwards (applying $70 \%$ of pushing power) and simultaneously guide the head downwards (applying 30\% of pushing power) (Fig. 2).

$d$. When the head is below the level of the fetal buttocks, direct the head securely into the maternal pelvis. This is to

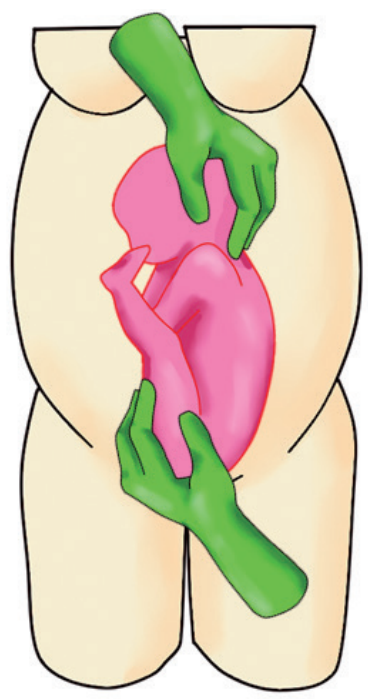

Fig. 1. Maneuver showing dislodgement of the fetal buttocks from the maternal pelvis. 


\section{Obstetrics \& Gynecology Science}

Gwang Jun Kim. External cephalic version

ensure that the fetal head does not revert to its original (breech) position (Fig. 3).

e. If the forward roll fails, a backward flip can be tried in the opposite direction of the fetal occiput descending first.

$f$. The ECV procedure can be performed either by a single operator or with the help of an assistant (to help with pushing the buttocks upward or with performing ultrasonography during the procedure).

g. Discontinue the procedure if the woman complains of significant discomfort or if the fetal heart rate is atypical or abnormal.

h. If the fetal heart rate does not recover within 3 minutes of emergency measures (maternal lateral position, bolus fluid infusion, and/or oxygen mask placement, among other such measures), it is necessary to prepare for an emergency cesarean section. If the fetal heart rate does not recover within 5 minutes, an emergency cesarean section is performed. Delivery should be completed within 10 minutes after the onset of bradycardia [22].

\section{Interventions and medications to increase the success rate of external cephalic version}

Various interventions have been used including the administration of tocolytics [55], neuraxial analgesia (epidural, spinal) [56], opioids, hypnosis, amnioinfusion, and the use of lubricants to increase the success rate of ECV. Recently, a Cochrane review of 28 studies reported that tocolytics (beta-

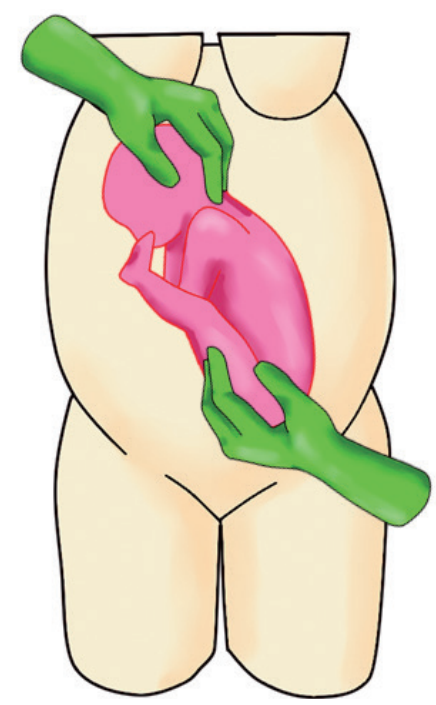

Fig. 2. Maneuver showing the operator pushing the fetal buttocks upward and guiding the fetal head. agonists) were significantly effective (relative risk, 1.68; 95\% $\mathrm{Cl}, 1.14-2.48)$ in facilitating successful ECV [57], and the effectiveness increased when these were used in combination with regional analgesia.

However, the result of adding regional analgesia was underpowered secondary to lack of cost-effectiveness. Notably, no difference was observed in cephalic presentation in labor [57].

Individual studies have reported a significantly higher success rate of ECV associated with the use of epidural anesthesia. However, these studies may have been biased by low overall ECV success rates or physician preferences [12]. Although routine use of regional analgesia or neuraxial blockade is not recommended for ECV, these may be considered during a repeat attempt or in women unable to tolerate ECV without analgesia [55]. Presently, other methods including the use of vibroacoustic stimulation, amnioinfusion, systemic administration of opioids, and hypnosis are not recommended owing to lack of evidence. In the author's hospital, only a beta-agonist (ritodrine) is used during ECV, and no analgesia or anesthesia is used.

\section{After external cephalic version}

Transient bradycardia is common (9-9.6\%) and occurs during or immediately following ECV $[43,45]$, possibly secondary to the vagal reflex when pressure is exerted on the fetal head. EFM commonly shows decreased baseline variability and

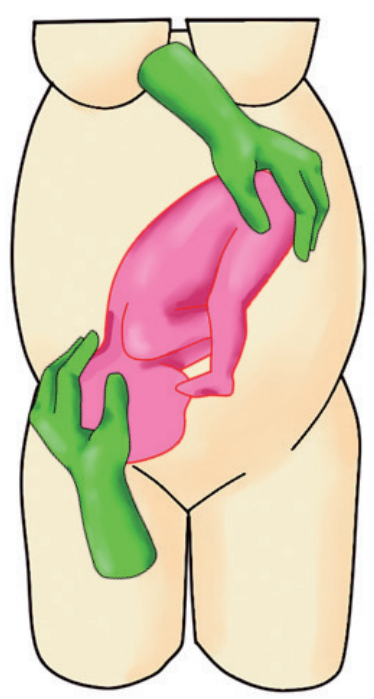

Fig. 3. Maneuver showing the operator directing the fetal head into the maternal pelvis. 


\section{Obstetrics \& Gynecology Science}

Vol. 62, No. 6, 2019

baseline heart rate, which represent the fetal stress response caused by decreased uteroplacental blood flow during the procedure [45]. It is recommended that EFM be continued for a minimum of 20 minutes after $E C V$, regardless of the success of the procedure.

\section{1) Warning for possible complications}

A woman undergoing ECV should be instructed to return promptly if she develops abdominal pain, symptoms of labor, bleeding, fluid leakage, fever, or decreased fetal movements.

\section{Management after external cephalic version}

\section{After successful external cephalic version}

Labor induction is not necessary after successful ECV, and maintaining the usual schedule of prenatal visits is recommended. Spontaneous reversion to breech presentation is not common and occurs in approximately $3 \%$ of cases [15].

\section{After external cephalic version failure}

The option of scheduling the next ECV trial (on another day) or a cesarean delivery can be discussed based on the woman's choice and fetal condition. The woman is also informed that $3-7 \%$ of term breech fetuses spontaneously turn to a cephalic presentation [58].

\section{Rh immunoglobulin injection}

Most authors recommend that $300 \mu \mathrm{g}$ of Rh immunoglobulin be injected in unsensitized Rh-negative women after an ECV trial. However, routine Rh immunoglobulin injection and performing a Kleihauer-Betke test are challenged by other authors because usually ECV-induced blood loss $>30 \mathrm{~mL}$ occurs only in $0.08 \%$ of women undergoing the procedure [48].

\section{Conclusion}

Currently, both the RCOG and ACOG recommend that all women with an uncomplicated singleton breech presentation at term should be offered an ECV trial $[11,12]$. It is known that ECV is a relatively simple and safe maneuver, which effectively reduces the risk of breech presentation at term. In Korea, the current trend involves performing a cesarean section in women with term breech presentation. If the situation is to change, it is important to overcome the main barrier to performing ECV among obstetricians-the lack of knowledge to educate and counsel pregnant women regarding ECV [14]. This review is a step in this direction and is aimed at providing obstetricians with the requisite knowledge to enable them to offer the benefit of this procedure to eligible women, to reduce the alarming increase in the rate of cesarean sections performed in Korea.

\section{Conflict of interest}

No potential conflict of interest relevant to this article was reported.

\section{Ethical approval}

No ethical approval was required for this study, which presents a literature-based review.

\section{Patient consent}

This being a review article, obtaining patient consent was not necessary.

\section{References}

1. Hofmeyr GJ, Kulier R, West HM. External cephalic version for breech presentation at term. Cochrane Database Syst Rev 2015:CD000083.

2. Martin JA, Hamilton BE, Osterman MJ, Driscoll AK, Drake P. Birth: final data for 2016. Natl Vital Stat Rep 2018;67:1-55.

3. Hannah ME, Hannah WJ, Hewson SA, Hodnett ED, Saigal $S$, Willan $A R$, et al. Planned caesarean section versus planned vaginal birth for breech presentation at term: a randomised multicentre trial. Lancet 2000;356:1375-83.

4. Martin JA, Hamilton BE, Sutton PD, Ventura SJ, Menacker F, Munson ML. Births: final data for 2002. Natl Vital Stat Rep 2003;52:1-113.

5. Organization for Economic Co-operation and Develop- 


\section{Obstetrics \& Gynecology Science}

Gwang Jun Kim. External cephalic version

ment. OECD health at a glance 2017: OECD indicators. Paris: OECD publishing; 2017.

6. World Health Organization. WHO statement on caesarean section rates. Geneva: WHO; 2015.

7. Gabay M, Molfe SM. Unnecessary cesarean sections: curing a national epidemic. Washington, D.C.: Public Citizen Health Research Group; 1994.

8. Tan JM, Macario A, Carvalho B, Druzin ML, El-Sayed YY. Cost-effectiveness of external cephalic version for term breech presentation. BMC Pregnancy Childbirth 2010;10:3.

9. American College of Obstetrics and Gynecologists. Clinical management guidelines for obstetrician-gynecologists number 13: external cephalic version. Washington, D.C.: ACOG; 2000.

10. Coco AS, Silverman SD. External cephalic version. Am Fam Physician 1998;58:731-8.

11. Royal College of Obstetricians and Gynecologists. External cephalic version and reducing the incidence of term breech presentation (green-top guideline No. 20a). London; RCOG: 2017.

12. American College of Obstetrics and Gynecologists. Practice bulletin No. 161: external cephalic version. Washington, D.C.: ACOG; 2016.

13. Rosman AN, Vlemmix F, Fleuren MA, Rijnders ME, Beuckens A, Opmeer BC, et al. Patients' and professionals' barriers and facilitators to external cephalic version for breech presentation at term, a qualitative analysis in the Netherlands. Midwifery 2014;30:324-30.

14. Zhang J, Bowes WA Jr, Fortney JA. Efficacy of external cephalic version: a review. Obstet Gynecol 1993;82:30612.

15. Collins S, Ellaway P, Harrington D, Pandit M, Impey LW. The complications of external cephalic version: results from 805 consecutive attempts. BJOG 2007;114:636-8.

16. Paul $C$. The baby is for turning: external cephalic version. BJOG 2017;124:773.

17. Bradley-Watson PJ. The decreasing value of external cephalic version in modern obstetric practice. Am J Obstet Gynecol 1975;123:237-40.

18. External cephalic version and reducing the incidence of term breech presentation: green-top guideline No. 20a. BJOG 2017;124:e178-92.

19. Hutton EK, Hofmeyr G, Dowswell T. External cephalic version of breech presentation before term. Cochrane
Database Syst Rev 2015:CD000084.

20. Ferguson JE 2nd, Dyson DC. Intrapartum external cephalic version. Am J Obstet Gynecol 1985;152:297-8.

21. Kaneti H, Rosen D, Markov S, Beyth $Y$, Fejgin MD. Intrapartum external cephalic version of footling-breech presentation. Acta Obstet Gynecol Scand 2000;79:1083-5.

22. Suyama F, Ogawa K, Tazaki Y, Miwa T, Taniguchi K, Nakamura $\mathrm{N}$, et al. The outcomes and risk factors of fetal bradycardia associated with external cephalic version. J Matern Fetal Neonatal Med 2019;32:922-6.

23. Sela HY, Fiegenberg T, Ben-Meir A, Elchalal U, Ezra Y. Safety and efficacy of external cephalic version for women with a previous cesarean delivery. Eur J Obstet Gynecol Reprod Biol 2009;142:111-4.

24. Burgos J, Cobos P, Rodríguez L, Osuna C, Centerno MM, Martínez-Astorquiza T, et al. Is external cephalic version at term contraindicated in previous cesarean section? A prospective comparative cohort study. BJOG 2014;121:230-5.

25. Rosman AN, Guijt A, Vlemmix F, Rijnders M, Mol BW, Kok M. Contraindications for external cephalic version in breech position at term: a systematic review. Acta Obstet Gynecol Scand 2013;92:137-42.

26. Ebner F, Friedl TW, Leinert E, Schramm A, Reister F, Lato $K$, et al. Predictors for a successful external cephalic version: a single centre experience. Arch Gynecol Obstet 2016;293:749-55.

27. El-Toukhy T, Ramadan G, Maidman D, Hanna L, Waterstone M. Impact of parity on obstetric and neonatal outcome of external cephalic version. J Obstet Gynaecol 2007;27:580-4.

28. Melo P, Georgiou EX, Hedditch A, Ellaway P, Impey L. External cephalic version at term: a cohort study of 18 years' experience. BJOG 2019;126:493-9.

29. Nassar N, Roberts CL, Cameron CA, Peat B. Outcomes of external cephalic version and breech presentation at term, an audit of deliveries at a Sydney tertiary obstetric hospital, 1997-2004. Acta Obstet Gynecol Scand 2006;85:1231-8.

30. Ben-Meir A, Erez Y, Sela HY, Shveiky D, Tsafrir A, Ezra Y. Prognostic parameters for successful external cephalic version. J Matern Fetal Neonatal Med 2008;21:660-2.

31. Kok M, van der Steeg JW, van der Post JA, Mol BW. Prediction of success of external cephalic version after 36 weeks. Am J Perinatol 2011;28:103-10. 


\title{
Obstetrics \& Gynecology Science
}

\author{
Vol. 62, No. 6, 2019
}

32. Boucher M, Bujold E, Marquette GP, Vezina Y. The relationship between amniotic fluid index and successful external cephalic version: a 14-year experience. Am J Obstet Gynecol 2003;189:751-4.

33. Kok M, Cnossen J, Gravendeel L, Van Der Post JA, Mol BW. Ultrasound factors to predict the outcome of external cephalic version: a meta-analysis. Ultrasound Obstet Gynecol 2009;33:76-84.

34. Haas DM, Magann EF, Haas DM, Magann EF. External cephalic version with an amniotic fluid index $<$ or $=$ 10: a systematic review. J Matern Fetal Neonatal Med 2005; 18:249-52.

35. Burgos J, Melchor JC, Cobos P, Centeno M, Pijoan $J I$, Fernandez-Llebrez L, et al. Does fetal weight estimated by ultrasound really affect the success rate of external cephalic version? Acta Obstet Gynecol Scand 2009;88:1101-6.

36. Velzel J, de Hundt M, Mulder FM, Molkenboer JF, Van der Post JA, Mol BW, et al. Prediction models for successful external cephalic version: a systematic review. Eur J Obstet Gynecol Reprod Biol 2015;195:160-7.

37. Chauhary S, Contag S, Yao R. The impact of maternal body mass index on external cephalic version success. J Matern Fetal Neonatal Med 2019;32:2159-65.

38. Hofmeyr GJ. Effect of external cephalic version in late pregnancy on breech presentation and caesarean section rate: a controlled trial. $\mathrm{Br} J$ Obstet Gynaecol 1983;90:392-9.

39. Lim PS, Ng BK, Ali A, Shafiee MN, Kampan NC, Mohamed Ismail NA, et al. Successful external cephalic version: factors predicting vaginal birth. ScientificWorldJournal 2014;2014:860107.

40. Burgos J, Iglesias M, Pijoan Jl, Rodriguez L, FernándezLlebrez L, Martínez-Astorquiza T. Probability of cesarean delivery after successful external cephalic version. Int J Gynaecol Obstet 2015;131:192-5.

41. de Hundt M, Vlemmix F, Bais JM, de Groot CJ, Mol BW, Kok M. Risk factors for cesarean section and instrumental vaginal delivery after successful external cephalic version. J Matern Fetal Neonatal Med 2016;29:2005-7.

42. de Hundt M, Velzel J, de Groot CJ, Mol BW, Kok M. Mode of delivery after successful external cephalic version: a systematic review and meta-analysis. Obstet Gynecol 2014;123:1327-34.

43. Kuppens SM, Smailbegovic I, Houterman S, de Leeuw
I, Hasaart TH. Fetal heart rate abnormalities during and after external cephalic version: which fetuses are at risk and how are they delivered? BMC Pregnancy Childbirth 2017; 17:363.

44. Collaris RJ, Oei SG. External cephalic version: a safe procedure? A systematic review of version-related risks. Acta Obstet Gynecol Scand 2004;83:511-8.

45. Hofmeyr GJ, Sonnendecker EW. Cardiotocographic changes after external cephalic version. Br J Obstet Gynaecol 1983;90:914-8.

46. Nassar N, Roberts CL, Barratt A, Bell JC, Olive EC, Peat B. Systematic review of adverse outcomes of external cephalic version and persisting breech presentation at term. Paediatr Perinat Epidemiol 2006;20:163-71.

47. Nassar N, Roberts CL, Cameron CA, Peat B. Outcomes of external cephalic version and breech presentation at term, an audit of deliveries at a Sydney tertiary obstetric hospital, 1997-2004. Acta Obstet Gynecol Scand 2006;85:1231-8.

48. Boucher M, Marquette GP, Varin J, Champagne J, Bujold E. Fetomaternal hemorrhage during external cephalic version. Obstet Gynecol 2008;112:79-84.

49. Grootscholten K, Kok M, Oei SG, Mol BW, van der Post JA. External cephalic version-related risks: a meta-analysis. Obstet Gynecol 2008;112:1143-51.

50. Tong Leung VK, Suen SS, Singh Sahota D, Lau TK, Yeung Leung T. External cephalic version does not increase the risk of intra-uterine death: a 17-year experience and literature review. J Matern Fetal Neonatal Med 2012;25:1774-8.

51. Balayla J, Dahdouh EM, Villeneuve S, Boucher M, Gauthier RJ, Audibert F, et al. Obstetrical and neonatal outcomes following unsuccessful external cephalic version: a stratified analysis amongst failures, successes, and controls. J Matern Fetal Neonatal Med 2015;28:605-10.

52. Son M, Roy A, Grobman WA, Miller ES. Association between attempted external cephalic version and perinatal morbidity and mortality. Obstet Gynecol 2018;132:36570.

53. Kirkinen P, Ylöstalo P. Ultrasonic examination before external version of breech presentation. Gynecol Obstet Invest 1982;13:90-7.

54. Donald WL, Barton JJ. Ultrasonography and external cephalic version at term. Am J Obstet Gynecol 1990;162:1542-5. 


\section{Obstetrics \& Gynecology Science}

Gwang Jun Kim. External cephalic version

55. Impey L, Pandit M. Tocolysis for repeat external cephalic version in breech presentation at term: a randomised, double-blinded, placebo-controlled trial. BJOG 2005;112:627-31.

56. Magro-Malosso ER, Saccone G, Di Tommaso M, Mele M, Berghella V. Neuraxial analgesia to increase the success rate of external cephalic version: a systematic review and meta-analysis of randomized controlled trials. Am J
Obstet Gynecol 2016;215:276-86.

57. Cluver C, Gyte GM, Sinclair M, Dowswell T, Hofmeyr GJ. Interventions for helping to turn term breech babies to head first presentation when using external cephalic version. Cochrane Database Syst Rev 2015:CD000184.

58. External cephalic version and reducing the incidence of term breech presentation: green-top guideline No. 20a. BJOG 2017;124:e178-92. 